\title{
The Sinorhizobium meliloti ExpE1 protein secreted by a type I secretion system involving ExpD1 and ExpD2 is required for biosynthesis or secretion of the exopolysaccharide galactoglucan
}

\author{
Leonilde M. Moreira,† Jörg D. Becker, Alfred Pühler and Anke Becker
} Author for correspondence: Anke Becker. Tel: +49521 106 5620. Fax: +49521 1065626.
e-mail: anke.becker@genetik.uni-bielefeld.de

Lehrstuhl für Genetik, Fakultät für Biologie, Universität Bielefeld, Postfach 100131, 33501 Bielefeld, Germany

\begin{abstract}
In Sinorhizobium meliloti the biosynthesis of the exopolysaccharide galactoglucan (EPS II) is directed by the exp genes. The expD1 and expD2 gene products are homologous to components of type I secretion systems. ExpE1, the gene of which is located adjacent to expD1 and expD2, was detected in S. meliloti cells and culture supernatants. ExpD1 and ExpD2 were required for the secretion of ExpE1, indicating that ExpE1 is secreted by a type I secretion system involving ExpD1 and ExpD2. ExpE1 contains 15 aspartate- and glycinerich nonapeptide repeats that were suggested to bind $\mathrm{Ca}^{2+}$. The ability to bind $\mathrm{Ca}^{2+}$ was demonstrated for a recombinant ExpE1 protein. Extracellular EPS II was not detected in cultures of non-polar expD1, expD2 and expE1 deletion mutants implying that these three genes are required for biosynthesis or secretion of galactoglucan.
\end{abstract}

Keywords: Rhizobium meliloti, exopolysaccharides, type I secretion, $\mathrm{Ca}^{2+}$-binding protein

\section{INTRODUCTION}

Bacterial exopolysaccharides (EPSs) are essential for the establishment of the nitrogen-fixing symbiosis between Sinorbizobium meliloti and the host plant Medicago sativa (Leigh \& Walker, 1994). Bacterial mutants unable to produce EPS induce the formation of root nodules on their host plant but fail to invade these nodules (González et al., 1996; Leigh \& Walker, 1994). Under standard conditions, S. meliloti wild-type strain SU47 and its streptomycin-resistant derivative strains 1021 and 2011 secrete an acidic EPS termed succinoglycan (EPS I). This EPS is composed of octasaccharide repeating units containing one galactose and seven glucose residues, and can be decorated by acetyl, succinyl and pyruvyl groups (Reinhold et al., 1994; Wang et al., 1999). The exo/exs gene cluster responsible

†Present address: Centro de Engenharia Biológica e Química, Instituto Superior Técnico, Av. Rovisco Pais, 1049-001 Lisboa, Portugal.

Abbreviations: ABC, ATP-binding cassette; EPS, exopolysaccharide; EPS I, succinoglycan; EPS II, galactoglucan; HMM, high molecular mass; LMM, low molecular mass; MBP, maltose-binding protein; MCS, multiple cloning site. for the biosynthesis of EPS I has been extensively studied and functions were proposed for most of the exo and exs gene products (Becker \& Pühler, 1998; Leigh \& Walker, 1994).

In addition, S. meliloti strains 1021 and 2011 have the ability to produce a second EPS, termed galactoglucan (EPS II), which can replace EPS I in nodule invasion (Glazebrook \& Walker, 1989; González et al., 1996; Zhan et al., 1989). EPS II is composed of alternating glucose and galactose residues which are decorated by acetyl and pyruvyl groups (Glazebrook \& Walker, 1989). Under standard culture conditions in complex media, large amounts of EPS I and almost no EPS II are produced. The production of EPS II was observed in the presence of a mutation in either of the regulatory genes $\operatorname{expR}$ (Glazebrook \& Walker, 1989) or mucR (Keller et al., 1995; Zhan et al., 1989). In addition, Zhan et al. (1991) showed that under phosphate-limiting conditions the wild-type strain 1021 produces EPS II. Extra copies of the exp gene cluster responsible for EPS II biosynthesis or extra copies of the transcriptional regulator $\exp G$ also resulted in the biosynthesis of EPS II (Glazebrook \& Walker, 1989; Rüberg et al., 1999; Zhan et al., 1989). 
The sequence of the exp gene cluster directing the biosynthesis of EPS II was previously reported and the inferred properties of the encoded gene products implied that these proteins are involved in regulation of exp gene expression, diphospho-nucleotide sugar biosynthesis, polymerization and export (Becker et al., 1997). Homologies of the deduced amino acid sequences of ExpD1 and ExpD2 to components of type I secretion systems implied that the $\mathrm{N}$-terminal domain of ExpD1 represents a membrane-spanning domain and the C-terminal portion is the ATP-binding cassette (ABC) domain (Becker et al., 1997). ExpD2 displays homologies to accessory factors of these secretion systems designated membrane fusion proteins (MFPs). Secretion complexes consisting of an ABC transporter and a MFP in conjunction with an outer-membrane protein are involved in the signal-peptide-independent secretion of proteins which usually contain a C-terminal secretion signal (Dinh et al., 1994). Most of these secreted proteins contain several aspartate- and glycine-rich nonapeptide tandem repeats involved in the binding of $\mathrm{Ca}^{2+}$ near the C-terminal secretion signal (Baumann, 1994; Economou et al., 1990; Ludwig et al., 1988).

Genes encoding type I secretion systems are usually situated adjacent to the genes encoding the secreted proteins (Fath \& Kolter, 1993). The protein encoded by $\operatorname{expE1}$, the gene for which is located immediately downstream of the expD1-expD2 operon, contains 15 aspartate- and glycine-rich nonapeptide repeats, and the structural characteristics of the C-terminal portion of ExpE1 are similar to those of C-terminal secretion signals (Becker et al., 1997). These features of ExpE1 suggest that this protein is a candidate to be secreted by a transporter complex involving ExpD1 and ExpD2. In this study we have analysed the role of expD1 and $\operatorname{expD} 2$ in secretion of ExpE1 and the involvement of the $\operatorname{expD1} \operatorname{expD2}$ and $\operatorname{expE1}$ genes in EPS II production.

\section{METHODS}

Microbiological methods. Bacterial strains and plasmids used in this study are listed in Table 1. Escherichia coli strains were grown in superbroth (Ausubel et al., 1990) or in LB medium (Sambrook et al., 1989) at $37^{\circ} \mathrm{C}$. S. meliloti strains were grown in TY (Beringer, 1974), LB or MOPS-buffered medium supplemented with $0 \cdot 1 \mathrm{mM}$ phosphate (Neidhardt et al., 1974) at $30^{\circ} \mathrm{C}$. Antibiotics were supplemented as required at the following concentrations $\left(\mu \mathrm{g} \mathrm{ml}^{-1}\right)$ : for $S$. meliloti, spectinomycin, 200; streptomycin, 600; nalidixic acid, 8; tetracycline, 8; gentamicin, 40; and neomycin, 120 ; for E. coli, tetracycline, 10; ampicillin, 100; gentamicin, 10; chloramphenicol, 50; and kanamycin, 50 .

DNA manipulations. Preparation of plasmid DNA, DNA restriction, agarose gel electrophoresis, cloning and transformation of E. coli cells were carried out according to established protocols (Sambrook et al., 1989). DNA was sequenced with the $\Delta \mathrm{Taq}$ cycle sequencing kit (Amersham), using Cy5-labelled primers and processed using the ALF DNA Express Sequencer (Pharmacia) according to the manufacturer's instructions. Southern hybridizations were performed as described by Kessler (1992). Total DNA from rhizobia was isolated as described by Meade et al. (1982).
Mutations were transferred between $S$. meliloti strains by phage $\Phi$ M12-mediated general transduction as described by Finan et al. (1984). Transfer of the mutations was verified by Southern hybridizations.

Construction of $\operatorname{expD1}$, expD2 and expE1 deletion mutants. The 7633 bp KpnI-EcoRI fragment of the exp gene cluster of S. meliloti 2011 (Becker et al., 1997) containing a deletion that ranged from nt 36 to $\mathrm{nt} 1756$ of the $1770 \mathrm{nt} \operatorname{expD1}$ coding region $\left(\operatorname{expD} 1_{\Delta 36-1756}\right)$ was cloned into the multiple cloning site (MCS) of suicide vector pK18mobG (Katzen et al., 1999). Start and stop codons of the remaining expD1 coding region were in-frame due to the presence of some nucleotides from the pK18mobG MCS. The same procedure was followed for the deletion of large parts of the expD2 and expE1 genes. The 4182 bp BglII-EcoRI fragment and the 6292 bp BalI-HindIII fragment of the exp gene cluster containing respectively, a deletion ranging from nt 37 to nt 1009 of the 1422 nt expD2 coding region $\left(\operatorname{expD} 2_{\Delta 37-1009}\right)$ and a deletion ranging from $n t 2$ to nt 616 of the $660 \mathrm{nt} \operatorname{expE1}$ coding region $\left(\exp E 1_{\Delta 2-616}\right)$ were inserted into the MCS of pK19mobG or pK18mobG. The plasmids obtained were designated pLM82-4, pLM87-1 and

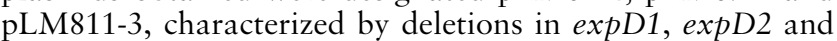
expE1, respectively. These plasmids were mobilized from strain E. coli S17-1 (Simon et al., 1983) to S. meliloti 2011. Homogenotization of these mutations was carried out as described by Katzen et al. (1999).

Plasmids pLM91-1 and pLM93-2 were obtained by insertion of a 2268 bp PvuII-EcoRI fragment containing expE1 under the control of its own promoter into the broad-host-range vector pSUP104 (Priefer et al., 1985) and suicide vector pK18mob (Schäfer et al., 1994), respectively. These plasmids were transferred to RmLM9835 and RmLM9836. Plasmid pLM811-1 was obtained by cloning a 3927 bp BalI fragment containing expD1 and expD2 under the control of the promoter of the expD operon into vector pK18mobG (Katzen et al., 1999). This plasmid was mobilized to SmLM9828 and SmLM9832.

To obtain plasmid pExpE7-lacZ a promoterless lacZ-Gm interposon (Becker et al., 1995) was inserted in sense orientation into the EcoRV site of expE7 cloned into pK18mob. This plasmid was homogenotized to Rm101 (Becker et al., 1997) and SmLM9909. These strains were grown until OD 600 $0.5-0.6$ in LB medium and assayed for $\beta$-galactosidase activity as described by Miller (1972).

All plasmid integrations into the genome and homogenotizations were verified by Southern hybridizations.

Purification of recombinant ExpE1. The 980 bp BalI fragment from pAB56-9 (Table 1) containing expE1 was inserted into the BamHI site of pWH844 (Schirmer et al., 1997) blunted by $\mathrm{S} 1$ nuclease. Correct fusion between the expE1 structural gene and the $(\mathrm{His})_{6}$-encoding region of $\mathrm{pWH} 844$ was verified by DNA sequencing. The resulting plasmid was designated pLM75-2 and transferred to E. coli SURE (Stratagene).

Purification of ExpE1 was performed using the QIAexpressionist protein purification system (Qiagen) with some modifications. After lysis, the cell-free extract was loaded onto a nickel-nitrilotriacetic acid (Ni-NTA) agarose column (Superflow) equilibrated in lysis buffer. The column was washed with 6 vols buffer A $(50 \mathrm{mM}$ Tris $/ \mathrm{HCl}, 300 \mathrm{mM}$ $\mathrm{NaCl}, 10 \%, \mathrm{v} / \mathrm{v}$, glycerin, $\mathrm{pH} 7 \cdot 5)$ and 6 vols buffer $\mathrm{B}(100 \mathrm{mM}$ Tris/ $\mathrm{HCl}, 500 \mathrm{mM} \mathrm{NaCl}, \mathrm{pH} \mathrm{8.9)}$. Bound proteins were eluted with buffer $\mathrm{B}$ by using a step gradient containing $0-0.5 \mathrm{M}$ imidazole. Proteins from collected fractions were visualized by vertical SDS-PAGE with $15 \%$ acrylamide (Laemmli, 1970) stained with Coomassie brilliant blue R250. 
Table 1. Strains, plasmids and phage

$\mathrm{Nx}$, nalidixic acid; Sm, streptomycin; Spc, spectinomycin; Tc, tetracycline; Km, kanamycin; Ap, ampicillin; Cm, cloramphenicol, Gm, gentamycin.

\begin{tabular}{|c|c|c|}
\hline $\begin{array}{l}\text { Strain/plasmid/ } \\
\text { phage }\end{array}$ & Relevant characteristics & Source/reference \\
\hline \multicolumn{3}{|l|}{ Strains } \\
\hline \multicolumn{3}{|l|}{ S. meliloti } \\
\hline Rm2011 & Wild-type, $\mathrm{Nx}^{\mathrm{r}} \mathrm{Sm}^{\mathrm{r}}$ & Casse et al. (1979) \\
\hline Rm101 & $\begin{array}{l}\mathrm{Rm} 2011, \mathrm{Spc}^{\mathrm{r}} \text { cassette of } \mathrm{pHP} 45 \Omega \text { inserted into the } \\
\text { Pmacl site of mucR (mucR101-spc) }\end{array}$ & Becker et al. (1997) \\
\hline RmAR9007 & Rm2011, exoY-lacZ/aacC1 & Keller et al. (1995) \\
\hline $\begin{array}{l}\text { RmAR9007 } \\
\text { TD101 }\end{array}$ & Rm2011, exoY-lacZ/aacC1 mucR-spc & I. Quester* \\
\hline SmLM8311 & RmAR9007 TD101, expA3::Tn5 & This work \\
\hline RmAR1157 & Rm2011, exoY::Tn5 expA1-lacZ/aacC1 & A. Roxlau* \\
\hline SmLM9806 & $\mathrm{Rm} 2011, \Delta \operatorname{expD1}$ & This work \\
\hline SmLM9829 & $\mathrm{Rm} 2011, \Delta \operatorname{expD2}$ & This work \\
\hline SmLM9833 & $\mathrm{Rm} 2011, \Delta \exp E 1$ & This work \\
\hline SmLM9815 & $\mathrm{Rm} 2011, \Delta \operatorname{expD1}$ exoY-lacZ/aacC1 & This work \\
\hline SmLM9831 & $\mathrm{Rm} 2011, \Delta \operatorname{expD2}$ exoY-lacZ/aacC1 & This work \\
\hline SmLM9835 & $\mathrm{Rm} 2011, \Delta \exp E 1$ exoY-lacZ/aacC1 & This work \\
\hline SmLM9909 & $\mathrm{Rm} 2011, \Delta \exp E 1$ mucR-spc & This work \\
\hline SmLM9901 & SmLM9835 carrying pLM91-1 & This work \\
\hline SmLM9903 & $\begin{array}{l}\text { SmLM9835 carrying pLM93-2 integrated into the } \\
\text { genome }\end{array}$ & This work \\
\hline SmLM9906 & SmLM9815 carrying pLM91-1 & This work \\
\hline SmLM9828 & $\mathrm{Rm} 2011, \Delta \operatorname{expD1}$ exo Y-lacZ/AaccC1 mucR-spc & This work \\
\hline SmLM9832 & $\mathrm{Rm} 2011, \Delta \operatorname{expD2}$ exoY-lacZ/aacC1 mucR-spc & This work \\
\hline SmLM9836 & $\mathrm{Rm} 2011, \Delta \operatorname{expE1}$ exoY-lacZ/aacC1 mucR-spc & This work \\
\hline SmLM9904 & $\begin{array}{l}\text { SmLM9836 with pLM93-2 integrated into the } \\
\text { genome upstream of the expE1 deletion }\end{array}$ & This work \\
\hline SmLM9905 & $\begin{array}{l}\text { SmLM9836 with pLM93-2 integrated into the } \\
\text { genome downstream of the expE1 deletion }\end{array}$ & This work \\
\hline SmLM9907 & $\begin{array}{l}\text { SmLM9828 carrying plasmid pLM811-1 integrated } \\
\text { into the genome }\end{array}$ & This work \\
\hline SmLM9908 & $\begin{array}{l}\text { SmLM9832 carrying plasmid pLM811-1 integrated } \\
\text { into the genome }\end{array}$ & This work \\
\hline \multicolumn{3}{|l|}{ E. coli } \\
\hline XL1-Blue & recA1 lac $\left[\mathrm{F}^{\prime}\right.$ proAB lac $\left.{ }^{\mathrm{q}} \mathrm{Z} \Delta \mathrm{M} 15 \operatorname{Tn} 10\left(\mathrm{Tc}^{\mathrm{r}}\right)\right]$ thi & Bullock et al. (1987) \\
\hline S17-1 & $\begin{array}{l}\text { E. coli } 294 \text {, thi } \mathrm{RP} 4-2-\mathrm{Tc}:: \mathrm{Mu}-\mathrm{Km}:: \mathrm{Tn} 7 \\
\text { chromosomally integrated }\end{array}$ & Simon et al. (1983) \\
\hline $\mathrm{DH} 5 \alpha$ & recA1, lacUi69, O80dlacZ $\Delta \mathrm{M} 15$ & BRL \\
\hline SURE & $\begin{array}{l}\mathrm{e} 14^{-}(\text {mcrA }) \Delta(\text { mcrCB-hsdSMR-mrr }) 171 \text { end } A 1 \\
\text { supE44 thi-1 gyrA96 relA1 lac recB recJ sbsC } \\
\text { umuC::Tn5 }\left(\mathrm{Km}^{\mathrm{r}}\right) \text { uvrC }\left[\mathrm{F}^{\prime} \text { proAB, lacl } \mathrm{Z} \Delta \mathrm{M} 15\right. \\
\left.\operatorname{Tn} 10\left(\mathrm{Tc}^{\mathrm{r}}\right)\right]\end{array}$ & Stratagene \\
\hline \multicolumn{3}{|l|}{ Plasmids } \\
\hline pAB56-9 & $\begin{array}{l}\text { pUK } 21 \text { containing a } 4 \cdot 1 \mathrm{~kb} K p n \mathrm{I}-H i n \mathrm{dIII} \text { fragment } \\
\text { of the exp gene cluster }\end{array}$ & This work \\
\hline pWH844 & pQE9 derivative, $\operatorname{lacl}^{\mathrm{q}}, \mathrm{Ap}^{\mathrm{r}}$ & Schirmer et al. (1997) \\
\hline pMAL-p & malE-(MCS)-lacZ $\alpha$ fusion, $l a c \mathrm{I}^{\mathrm{q}}, \mathrm{Ap}^{\mathrm{r}}$ & New England Biolabs \\
\hline $\mathrm{pK} 18 / 19 \mathrm{mob}$ & pUC18/19 derivative, $\operatorname{lac} Z \alpha$, mob, $\mathrm{Km}^{\mathrm{r}}$ & Schäfer et al. (1994) \\
\hline pK18/19mobG & pK18/19mob derivative, gusA & Katzen et al. (1999) \\
\hline pSUP104 & $\begin{array}{l}\text { Broad-host-range plasmid pACYC184 derivative, } \\
m o b, \mathrm{Tc}^{\mathrm{r}}, \mathrm{Cm}^{\mathrm{r}}\end{array}$ & Priefer et al. (1985) \\
\hline
\end{tabular}


Table 1 (cont.)

\begin{tabular}{|c|c|c|}
\hline $\begin{array}{l}\text { Strain/plasmid/ } \\
\text { phage }\end{array}$ & Relevant characteristics & Source/reference \\
\hline pLM82-4 & $\begin{array}{l}\text { pK18mobG containing a } 7633 \text { bp KpnI-EcoRI } \\
\text { fragment from exp region with a } 1720 \text { nt } \operatorname{expD} 1 \\
\text { deletion }\end{array}$ & This work \\
\hline pLM87-1 & $\begin{array}{l}\text { pK19mobG containing a } 4182 \text { bp } B g l \mathrm{II}-E c o \mathrm{RI} \\
\text { fragment from exp region with a } 972 \text { nt } \operatorname{expD2} \\
\text { deletion }\end{array}$ & This work \\
\hline PLM811-3 & $\begin{array}{l}\text { pK18mobG containing a } 6292 \text { bp Ball-HindIII } \\
\text { fragment from exp region with a } 614 \text { nt } \operatorname{expE1} \\
\text { deletion }\end{array}$ & This work \\
\hline pLM91-1 & $\begin{array}{l}\text { pSUP104 containing a } 2268 \text { bp PvuII-EcoRI } \\
\text { fragment with the expE1 gene under the control } \\
\text { of its own promoter }\end{array}$ & This work \\
\hline pLM93-2 & 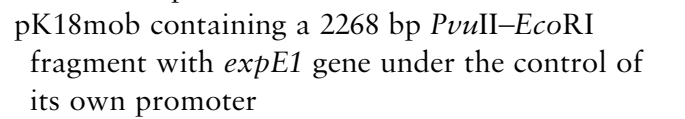 & This work \\
\hline pLM811-1 & $\begin{array}{l}\text { pK18mobG containing a } 3927 \text { bp BalI fragment } \\
\text { with } \operatorname{expD} 1 \text { and } \operatorname{expD} 2 \text { genes under the control } \\
\text { of the } \operatorname{expD} \text { operon promoter }\end{array}$ & This work \\
\hline pLM76-4 & $\begin{array}{l}258 \mathrm{nt} \text { of the } 3^{\prime} \text { terminus of the } \operatorname{expE1} \text { coding } \\
\text { region cloned into the } S t u \mathrm{I} \text { site of the pMAL-p } \\
\text { expression vector }\end{array}$ & This work \\
\hline pLM76-2 & $\begin{array}{l}\text { pMAL-p containing the complete expE1 gene } \\
\text { cloned in the StuI site }\end{array}$ & This work \\
\hline pLM75-2 & 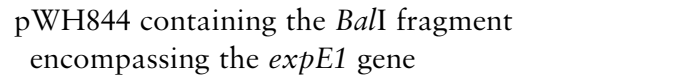 & This work \\
\hline pLM83-1 & $\begin{array}{l}\text { pWH844 derivative containing the complete expE1 } \\
\text { gene }\end{array}$ & This work \\
\hline pLM82-6 & $\begin{array}{l}\text { pK19mob expressing a translational fusion } \\
\text { ExpE1-LacZ }\end{array}$ & This work \\
\hline pExpE7-lacZ & $\begin{array}{l}\text { Promoterless lacZ-Gm interposon inserted into } \\
\text { the EcoRV site of the expE7 coding region }\end{array}$ & Becker et al. (1997) \\
\hline \multicolumn{3}{|l|}{ Phage } \\
\hline ФM12 & S. meliloti transducing phage & Finan et al. (1984) \\
\hline
\end{tabular}

*Lehrstuhl für Genetik, Universität Bielefeld, Germany.

The most purified fractions of (His) ${ }_{6}$ ExpE1 were pooled, concentrated in an Ultrafree Concentrator (molecular mass cutoff, $10 \mathrm{kDa}$; Millipore) and then separated on a Sephacryl S200 HR column (16 mm diameter, $600 \mathrm{~mm}$ length) according to the manufacturer's recommendations (Pharmacia) with buffer B as the mobile phase. The eluted (His) ${ }_{6}$ ExpE1 was associated with a major protein peak as confirmed by SDSPAGE. The purification of (His) ${ }_{6}$ ExpE1 to electrophoretic homogeneity was achieved by preparative gel electrophoresis (model 491 Prep Cell; Bio-Rad) according to the manufacturer's instructions. N-terminal protein sequencing was performed by the Max-Plank-Institut for Molecular Physiology.

Affinity purification of anti-ExpE1 antibodies. The pure ExpE1 containing the (His) ${ }_{6}$ tag was used for immunization of rabbits (Eurogentec). The serum obtained was subjected to immunoaffinity purification using the $85 \mathrm{C}$-terminal amino acids from ExpE1 fused in-frame to the maltose-binding protein (MBP). To obtain this fusion protein, the last $258 \mathrm{nt}$ of expE1 were amplified by PCR (upper primer 5'-GGCAGCGATATCTTCGTT-3'; lower primer 5' -CTATCAGTGGACAGTGAAGTAGTT-3') and cloned into the StuI site of vector pMAL-p (New England Biolabs) resulting in pLM76-4. Purification of MBP-ExpE1 $1_{\mathrm{C} \text {-term }}$ was performed according to a standard protocol (New England Biolabs) with some minor modifications. The osmotic-shock fluid containing the fusion protein was loaded onto an amylose resin column and the buffers used in the washing steps did not include Tween 20. The eluted protein was applied to a SDS-PAGE and electroblotted onto a nitrocellulose membrane. After protein staining with Ponceau $S$, the band corresponding to the $\mathrm{MBP}-\mathrm{ExpE} 1_{\mathrm{C} \text {-term }}$ fusion was removed and used as an immobilized antigen. Antibody affinity purification was performed as described by Harlow \& Lane (1988).

${ }^{45} \mathrm{Ca}^{2+}$ binding. To test the calcium-binding activity of ExpE1, 
the corresponding gene was amplified by PCR (upper primer 5'-ATGGCCACTTTGGAAGG-3' ; lower primer 5'-CTATCAGTGGACAGTGAAGTAGTT- $3^{\prime}$ ) and cloned in-frame with the MBP gene from pMAL-p, producing pLM76-2. This fusion protein (MBP-ExpE1) was purified by affinity chromatography as described above. MBP was cleaved from ExpE1 by protease factor $\mathrm{Xa}\left(2 \mathrm{~d}\right.$ at $\left.4{ }^{\circ} \mathrm{C}\right)$ resulting in the removal of vector-derived residues attached to ExpE1. The protein mixture containing ExpE1 was subjected to SDSPAGE, transferred onto a nitrocellulose membrane and treated as described by Maruyama et al. (1984) to identify $\mathrm{Ca}^{2+}$ binding proteins.

Immunodetection of proteins. Proteins separated by SDSPAGE were electroblotted onto nitrocellulose membranes by the procedure of Towbin et al. (1979). Immunodetection was performed according to standard protocols (Harlow \& Lane, 1988).

Analysis of ExpE1 in cell extract and culture supernatant. $S$. meliloti strains in the late-exponential growth phase $\left(\mathrm{OD}_{600}\right.$ $1 \cdot 5-2 \cdot 0)$ were grown in MOPS-buffered medium supplemented with $0.1 \mathrm{mM}$ phosphate. Cells were centrifuged for $5 \mathrm{~min}$ at $18000 \mathrm{~g}$ at $4{ }^{\circ} \mathrm{C}$ and the pellets were solubilized in SDS sample buffer to yield a preparation of total cellular proteins. Culture supernatants were separated from the cells by centrifugation $\left(20000 \mathrm{~g}, 4{ }^{\circ} \mathrm{C}, 30 \mathrm{~min}\right)$ and a protease-inhibitor cocktail (EDTA free; Boehringer) was added. A volume of $8 \mathrm{ml}$ of each supernatant was concentrated by filtration (Ultrafree Concentrator) and mixed with SDS sample buffer. Preparation of outer-membrane proteins from $S$. meliloti was performed according to a protocol described by Poxton et al. (1985). Protein concentrations were determined by using the Bio-Rad Protein Assay with BSA as a standard.

Analysis of extracellular carbohydrates in culture supernatants. S. meliloti strains were grown at $30^{\circ} \mathrm{C}$ for $10 \mathrm{~d}$ in MOPS-buffered medium supplemented with $0.1 \mathrm{mM}$ phosphate. Cells were removed by centrifugation $(20000 \mathrm{~g}, 1 \mathrm{~h})$ and the culture supernatants were desalted by dialysis (molecular mass cutoff $1 \mathrm{kDa}$ ) against water for $4 \mathrm{~d}$, followed by EPS concentration by lyophilization. High- (HMM) and lowmolecular-mass (LMM) fractions were separated by gelfiltration chromatography (twice on GFC 4000-8 and once on GFC $300-8$, column size $300 \times 7.7 \mathrm{~mm}$; Machery-Nagel; flow rate, $0.8 \mathrm{ml} \mathrm{min}^{-1} ; 200 \mathrm{mM}$ sodium chloride, $200 \mathrm{mM}$ sodium phosphate buffer, $\mathrm{pH} 7 \cdot 0$ ). Fractions were detected using a differential refraction index detector. Total carbohydrates were quantified by the $\mathrm{HCl} / \mathrm{L}$-cysteine method (Chaplin \& Kennedy, 1986). Glucose and galactose content of the HMM and LMM fractions was quantified by enzymic assays after hydrolysis with hydrochloric acid and subsequent neutralization (Beutler, 1984; Kunst et al., 1984).

\section{RESULTS}

An antiserum directed against the C-terminal portion of ExpE1 comprising 85 amino acids is specific for the ExpE1 protein

A (His) ${ }_{6}$ ExpE1 fusion protein was expressed from pLM75-2 in E. coli SURE (Fig. 1a, lane 1). SDS-PAGE of the protein purified by affinity chromatography exhibited a major band migrating at approximately $34 \mathrm{kDa}$ (Fig. 1a, lane 2), which reacted with anti-His-tag antibody (data not shown), but whose mass did not correspond to the calculated molecular mass of the $\mathrm{N}$ -
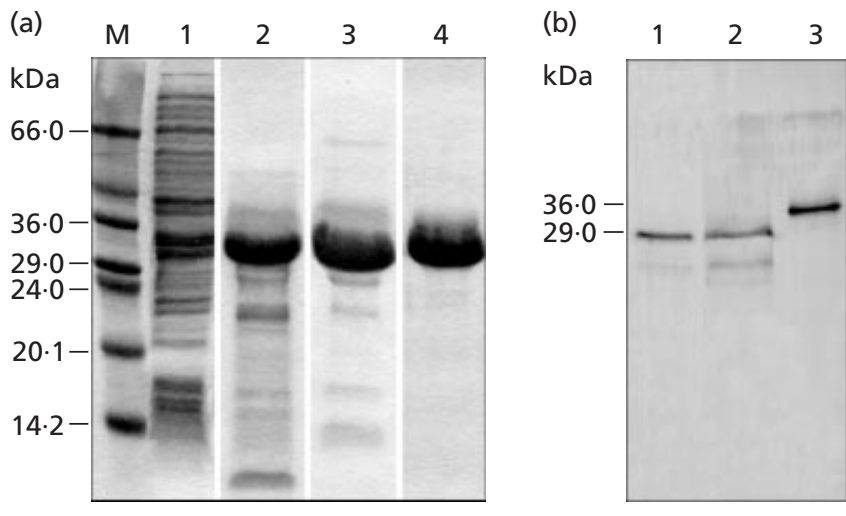

Fig. 1. (a) Purification of (His) ${ }_{6} \operatorname{ExpE} 1$. Coomassie blue-stained SDS-PAGE gel displaying the molecular mass marker Dalton VII-L (M); cell extract of E. coli SURE producing (His) ${ }_{6} \operatorname{ExpE} 1$ (from pLM75-2) grown in the presence of 1 mM IPTG (lane 1); sample of the peak fraction eluted from Ni-NTA affinity chromatography (lane 2); sample of the peak fraction after gel filtration (lane 3 ); purified (His) ${ }_{6} \operatorname{ExpE} 1$ ( $3 \mu \mathrm{g}$ protein) after preparative gel electrophoresis separation (lane 4). (b) Western blot analysis of ExpE1 in total-cell proteins of $S$. meliloti SmLM8311 (lane 1), E. coli SURE producing ExpE1 (pLM83-1) (lane 2) and the pure (His) ${ }_{6} \operatorname{ExpE} 1$ (lane 3), using anti-ExpE1 $1_{\text {C-term }}$ polyclonal antibodies.

terminal histidine-tagged ExpE1 (23240 Da). Further purification was achieved by size-fractionation chromatography (Fig. 1a, lane 3). The $M_{\mathrm{r}}$ of the native (His) ${ }_{6}$ ExpE1 was estimated to be approximately $46 \mathrm{kDa}$ (data not shown). Since the predicted $M_{\mathrm{r}}$ based on the DNA sequence is $23 \cdot 2 \mathrm{kDa}$, it is likely that the native protein purifies as a dimer. The essentially pure (His) ${ }_{6}$ ExpE1 protein that was obtained by preparative gel electrophoresis migrated as a single $34 \mathrm{kDa}$ band on a Coomassie blue-stained SDS-PAGE gel (Fig. 1a, lane 4). This protein was found to have an N-terminal 20 aa sequence that perfectly matched the predicted amino acid sequence of the engineered His-tagged ExpE1.

A polyclonal antiserum raised against the purified $(\text { His })_{6}$ ExpE1 detected the recombinant protein in total protein extracts from the E. coli expression strain. (His) ${ }_{6}$ ExpE1 could be detected with an antiserum dilution ranging from $1: 1000$ to $1: 6000$. Similar experiments were carried out in $S$. meliloti showing that this polyclonal antibody detected ExpE1 but also other proteins. No signal for the $S$. meliloti cell extract was detected with the preimmune serum. To overcome this lack of specificity, the polyclonal antibody was subjected to immunoaffinity purification using the 85 aa of the Cterminal part of ExpE1. This C-terminal region was chosen because ExpE1 contains 15 aspartate- and glycine-rich nonapeptide repeats in the $124 \mathrm{~N}$-terminal aa and none in the last 85 aa (Becker et al., 1997). Similar aspartate- and glycine-rich repeats, which might be recognized by the anti-(His) ${ }_{6}$ ExpE1 antiserum, were also identified in the ExsH and EglC proteins from $S$. meliloti (Sharypova et al., 1999; York \& Walker, 1997) and might also be present in additional S. meliloti 
(a)

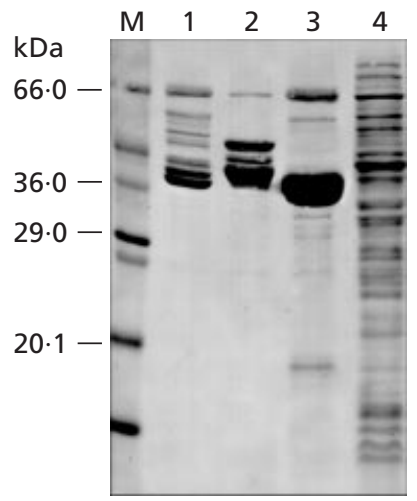

(b)

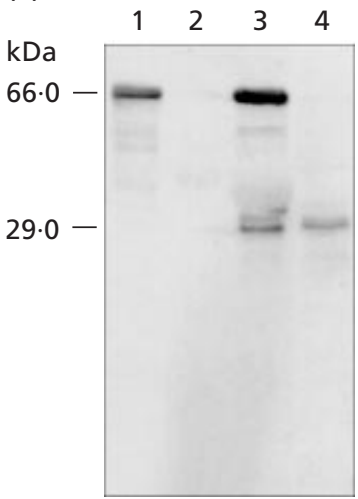

Fig. 2. Calcium-binding activity of ExpE1. ExpE1 protein obtained after cleavage of the MBP-ExpE1 fusion with factor Xa (lanes 3 ) or expressed by E. coli carrying pLM83-1 (lanes 4) was separated by SDS-PAGE and electroblotted. The protein fraction eluted from the amylose affinity chromatography containing MBP-ExpE1 expressed by pLM76-2 (lanes 1) and MBP expressed by pMAL-p (lanes 2) were included as controls. (a) Proteins stained with Ponceau S. (b) ExpE1 detection by autoradiography after incubation with ${ }^{45} \mathrm{Ca}^{2+}$. Lane $\mathrm{M}$ is marker Dalton VII-L.

proteins. Incubation of the purified immobilized MBPExpE1 $1_{\text {C-term }}$ fusion protein with the polyclonal antibody anti-(His) ${ }_{6}$ ExpE1 resulted in the purified serum antiExpE1 $1_{\text {C-term }}$ that reacted exclusively with the ExpE1 protein from S. meliloti cell extracts (Fig. 1b, lane 1), and detected ExpE1 in E. coli cell extracts as well as the purified (His) ${ }_{6}$ ExpE1 protein (Fig. 1b, lanes 2 and 3). Some additional signals were caused by degradation fragments of ExpE1, which migrated faster in the gel (Fig. 1b, lanes 1 and 2).

As described for (His) ${ }_{6}$ ExpE1, ExpE1 expressed in E. coli or S. meliloti also migrated abnormally in SDSPAGE. ExpE1 has a predicted $M_{\mathrm{r}} 22117$, but it migrated at approximately $30 \mathrm{kDa}$ (Fig. $1 \mathrm{~b}$, lanes 1 and 2).

\section{ExpE1 binds $\mathrm{Ca}^{2+}$}

Since ExpE1 contains 15 aspartate- and glycine-rich nonapeptide repeats, the ability of ExpE1 to bind $\mathrm{Ca}^{2+}$ was assayed. The (His) ${ }_{6}$ ExpE1 protein could not be used for this assay due to the capacity of the histidine tag to bind divalent cations, therefore the expE1 gene was expressed as a fusion with MBP in E. coli SURE. The $E$. coli cell extract was subjected to affinity chromatography and we observed, after SDS-PAGE, that beside the fusion protein MBP-ExpE1 at $64 \mathrm{kDa}$, another intense band running at approximately $42 \mathrm{kDa}$ was present (Fig. 2a, lane 1) that possibly represented the MBP alone. Such an observation was also reported by Maina et al. (1988) and can be explained by premature termination or cleavage of the fusion protein. Incubation of this MBP-ExpE1 eluted fraction with the protease factor Xa resulted in the release of ExpE1. This

cleavage gave a $42 \mathrm{kDa}$ band corresponding to the released $\mathrm{MBP}$ and a $30 \mathrm{kDa}$ band corresponding to ExpE1 (Fig. 2a, lane 3). Due to premature termination or cleavage of the fusion protein and some degradation of ExpE1, the concentration of MBP was higher than that of ExpE1 (Fig. 2a, lane 3). Since the cleavage with factor $\mathrm{Xa}$ was not complete, a $64 \mathrm{kDa}$ band corresponding to the non-cleaved MBP-ExpE1 was still present (Fig. 2a, lane 3). The eluted proteins together with protein cell extract from E. coli expressing the expE1 gene were tested for binding of ${ }^{45} \mathrm{Ca}^{2+}$. A band migrating at approximately $64 \mathrm{kDa}$ that corresponded to the fusion protein MBP-ExpE1 (Fig. 2b, lanes 1 and 3) and a band migrating at $30 \mathrm{kDa}$ representing ExpE1 (Fig. 2b, lane 3) was detected due to binding of ${ }^{45} \mathrm{Ca}^{2+}$. In this assay, ExpE1 was also detected in the protein cell extract from E. coli expressing the native ExpE1 protein (Fig. 2b, lane 4) but no signal was obtained from E. coli cell extract lacking the expE1 gene (data not shown). The observation that in Fig. 2b, lane 1, we obtain a radioactive signal only for the MBP-ExpE1 fusion but no signal with the cell extract in which MBP was expressed alone (Fig. 2b, lane 2), indicates that the binding of $\mathrm{Ca}^{2+}$ is due to ExpE1 and not to other E. coli proteins that may comigrate with ExpE1.

\section{ExpE1 is secreted by a transporter system involving ExpD1 and ExpD2}

To investigate the involvement of the $\operatorname{expD} 1, \operatorname{expD} 2$ and $\operatorname{expE1}$ genes in ExpE1 protein secretion and EPS II production, chromosomal mutants were generated using a gene-replacement strategy, whereby large parts of each of these genes were deleted giving rise to non-polar mutants. The deletion in expD1 extended from nt 36 to nt 1756 and the remaining sequence that contained some nucleotides from the vector encoded a peptide of 31 aa. To mutate $\operatorname{expD2}$, a fragment ranging from nt 37 to nt 1009 was deleted. The remaining sequence that included some nucleotides from the vector coded for a protein of 169 aa. The deletion from nt 2 to nt 616 of expE1 resulted in a remaining sequence that contained some nucleotides from the vector and encoded a peptide of 22 aa.

Under standard conditions, Rm2011 produces EPS I and only traces of EPS II, if any. However, in a mucR or $\exp R$ background and under phosphate-limiting conditions, synthesis of EPS II is stimulated (Glazebrook \& Walker, 1989; Keller et al., 1995; Zhan et al., 1991). To overexpress the exp genes leading to the biosynthesis of EPS , the mucR101-spc mutation (Becker et al., 1997) was transferred to each of the deletion mutants. To block biosynthesis of EPS $\mathrm{I}$, a lacZ-aacC1 insertion in the exoY gene (Keller et al., 1995) encoding the galactose-1-phosphate transferase that catalyses the first step in the synthesis of the EPS I subunit (Reuber \& Walker, 1993), was additionally transferred to the deletion mutants. The strains obtained were designated SmLM9815 (Rm2011 sexpD1exo Y), SmLM9828 (Rm2011 $\Delta \operatorname{expD1exoYmucR),~SmLM9831~(Rm2011~}$ 


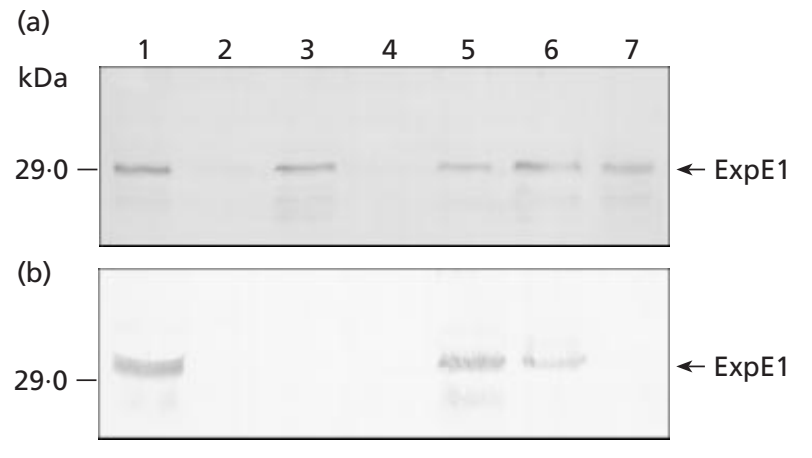

Fig. 3. Western blot analysis of ExpE1 in total-cell proteins (a) and supernatants (b) of $S$. meliloti strains using anti-ExpE $1_{C \text {-term }}$ polyclonal antibody. Lanes contain either the cells (without supernatant) from the equivalent of $10 \mu \mathrm{l}$ culture $\left(\mathrm{OD}_{600}\right.$ 1.5-2.0) (a) or $2 \mathrm{ml}$ cell-free concentrated supernatants (b). RmAR1157 (lanes 1), SmLM9815 (lanes 2), SmLM9831 (lanes 3), SmLM9835 (lanes 4), SmLM9901 (lanes 5), SmLM9903 (lanes 6) and SmLM9906 (lanes 7) are shown. Arrows indicate the position of the protein band corresponding to ExpE1.

$\Delta \operatorname{expD2exoY),~SmLM9832~(Rm2011~} \Delta \operatorname{expD2exoY-~}$ mucR), SmLM9835 (Rm2011 $\Delta$ expE1exo Y) and SmLM9836 (Rm2011 $\Delta$ expE1exoYmucR) (Table 1). Blocking EPS I and EPS II production was necessary to enable the high-efficiency removal of cells from culture supernatants and to facilitate the concentration of proteins. Therefore, strains RmAR1157 (Rm2011 expA1exoY) and SmLM8311 (Rm2011 expA3exoYmucR) were used to determine if ExpE1 is secreted. Detection of ExpE1 in the supernatant of $S$. meliloti strains was additionally complicated by proteolysis. Several bands migrating at sizes lower than $30 \mathrm{kDa}$ reacted with the antiExpE1 $1_{\text {C-term }}$ antibody (data not shown). This effect was enormously reduced by adding a protease-inhibitor cocktail to the culture supernatant immediately after removal of the cells.

RmAR1157 grown under phosphate-limiting conditions was assayed for the presence of ExpE1 in cells and supernatant using the anti-ExpE1 $1_{\mathrm{C}-\text { term }}$ antibody. The result indicated that ExpE1 is present both in the cell extract (Fig. 3a, lane 1) and the supernatant (Fig. 3b, lane 1). This implies that ExpE1 is secreted into the growth medium.

Some proteins like endoglycanases and cellulases from Rhizobium leguminosarum and Azorhizobium caulinodans secreted by type I secretion systems were found to be cell-surface associated (Geelen et al., 1995; Mateos et al., 1992). Therefore, we investigated whether ExpE1 can also become attached to the outer membrane of the cell. Preparation of outer-membrane proteins from RmAR9007 TD101 (Rm2011exoYmucR) was carried out according to the protocol described by Poxton et al. (1985). Using the anti-ExpE1 $1_{\mathrm{C} \text {-term }}$ antibody ExpE1 was not detected in the outer-membrane fraction, but in the cytoplasmic fraction (data not shown).

Since ExpE1 is predicted to be secreted via a signal peptide-independent pathway, it was tested whether this protein is secreted via a transport system involving ExpD1 and ExpD2 that display homologies to components of type I protein-secretion complexes (Becker et al., 1997). The double mutants SmLM9815 (Rm2011 $\Delta \operatorname{expD1exoY)}$ and SmLM9831 (Rm2011 $\Delta \operatorname{expD2exoY)}$ grown under phosphate-limiting conditions were tested for the presence of ExpE1 in cells and supernatants. Immunodetection showed the absence of ExpE1 in the growth medium (Fig. 3b, lanes 2 and 3 ) of both mutants, indicating that ExpE1 is secreted by a transporter system involving ExpD1 and ExpD2. The protein was found in the cell extract of SmLM9831 ( $\mathrm{Rm} 2011$ sexpD2exoY) (Fig. 3a, lane 3), but curiously only a very weak signal at the detection limit was observed in the cell extract of SmLM9815 (Rm2011 sexpD1exoY) (Fig. 3a, lane 2). The $\beta$-galactosidase activity of a translational expE1lac $Z$ fusion expressed in the wild-type background was comparable to the values obtained in the expD1 or $\operatorname{expD2}$ mutant. This implies that the deletions in expD1 and $\operatorname{expD2}$ did not influence the transcription or translation of expE1. ExpE1 was not detected in the cells or the supernatant of SmLM9835 (Rm2011 $\Delta \operatorname{expE1-~}$ exoY) (Fig. 3a and 3b, lanes 4).

To restore the ability of SmLM9835 to express ExpE1, pLM91-1 and pLM93-2 carrying the expE1 gene under the control of its own promoter were transferred to SmLM9835. Strain SmLM9901 contained the replicating plasmid pLM91-1 whereas strain SmLM9903 carried plasmid pLM93-2 integrated into the genome. The ExpE1 protein was detected in cell extracts and culture supernatants of both strains (Fig. 3a, b, lanes 5 and 6). The introduction of the multicopy plasmid pLM91-1 in SmLM9815 (SmLM9906) resulted in the detection of ExpE1 in the cell extract, but not in the supernatant (Fig. $3 a, b$, lanes 7). Since a very small amount of ExpE1 protein was detected in the cell fraction of SmLM9815, accumulation of ExpE1 in this mutant might only occur if it is overexpressed. Secretion of ExpE1 was also assayed in the deletion mutants carrying additionally an exoY mutation and a mucR mutation that stimulated the expression of the exp genes. The results of ExpE1 detection in these triple mutants were consistent with those described above, with the exception of strain SmLM9828 (Rm2011 sexpD1exoYmucR), in which ExpE1 was detected in the cells (data not shown).

\section{ExpD1, ExpD2 and ExpE1 are required for the synthesis or secretion of EPS II}

To study the involvement of the secreted protein ExpE1 and its transport system in EPS II secretion, S. meliloti strains were used that carried mutations in exoY and mucR and were grown in low-phosphate medium. Under these conditions, exp genes are maximally expressed (Rüberg et al., 1999). Using gel-filtration chromatography, HMM and LMM fractions present in the culture supernatants of strains carrying deletions in $\operatorname{expE1}$, expD1 and $\operatorname{expD2}$ were separated. No HMM fraction was detected in the culture supernatants of 
Table 2. Analysis of culture supernatants by gel-filtration chromatography and sugar content

Standard deviations were equal to or less than $10 \%$ and $4 \%$ for the determination of the total amount of extracellular carbohydrates in glucose equivalents and the ratio of glucose to galactose, respectively. ND, Not determined.

\begin{tabular}{|c|c|c|c|c|c|}
\hline \multirow[t]{2}{*}{ Strain } & \multirow{2}{*}{$\begin{array}{l}\text { Glucose } \\
\text { equivalents } \\
\left(\mathrm{mg} \mathrm{l}^{-1}\right)\end{array}$} & \multicolumn{2}{|c|}{ HMM fraction } & \multicolumn{2}{|c|}{ LMM fraction } \\
\hline & & Present? & Glc/Gal (\%) & Present? & Glc/Gal (\%) \\
\hline RnAR9007 TD101 & 397 & + & $51 / 49$ & + & ND \\
\hline SmLM9828 & 48 & - & $\mathrm{ND}$ & + & $100 / 0$ \\
\hline SmLM9832 & 534 & - & ND & + & $100 / 0$ \\
\hline SmLM9836 & 44 & - & ND & + & $100 / 0$ \\
\hline SmLM9904 & 312 & + & $51 / 49$ & + & ND \\
\hline SmLM9905 & 320 & + & $52 / 48$ & + & ND \\
\hline SmLM9907 & 435 & + & $53 / 47$ & + & ND \\
\hline SmLM9908 & 442 & + & $54 / 46$ & + & ND \\
\hline SmLM8311 & 17 & - & ND & + & $100 / 0$ \\
\hline SmLM8311/SmLM9836* & 31 & - & ND & + & $100 / 0$ \\
\hline
\end{tabular}

*Co-cultivation experiment. Values given refer to both strains together.

$\operatorname{expE1} 1, \operatorname{expD1}$ and $\operatorname{expD2}$ deletion mutants indicating the absence of extracellular HMM-EPS II (Table 2).

To exclude the possibility of a polar effect of the expE1 deletion on the transcription of the expE genes located downstream of expE1 (Becker et al., 1997), a promoterless lacZ-Gm interposon (Becker et al., 1995) was inserted into the expE7 gene of mutant SmLM9909

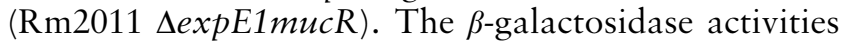
displayed by the resulting strain and the control strain carrying the expE7-lacZ transcriptional fusion and a mutation in mucR were comparable. Since promotermapping experiments indicated that expE7 is exclusively transcribed from a promoter upstream of expE1 (Becker et al., 1997), this result implies that the expE1 deletion had no influence on the transcription of the expE operon in the mucR mutant background. To confirm that the absence of HMW EPS II in SmLM9836 (Rm2011

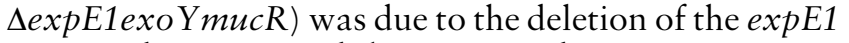
gene and not caused by a second-site mutation, a complementation strategy was used in which pLM93-2 containing expE1 was integrated into the genome of SmLM9836 (Rm2011 $\Delta \operatorname{expE1exoYmucR)~by~a~single~}$ homologous recombination event. Depending on whether the recombination occurred upstream or downstream of the expE1 deletion, transcription of the whole $\operatorname{expE}$ operon was driven by the expE1 promoter located in front of the expE1 deletion (SmLM9904) or in front of the expE1 wild-type gene (SmLM9905), respectively. Strains with both possible genomic structures regained the ability to produce HMW EPS II, providing additional evidence that the $\operatorname{expE1}$ deletion had no influence on the expression of the expE operon. Using lacZ transcriptional fusions no vector promoter that might have contributed to the transcription of the expE operon in the merodiploid strains was identified. Complemen- tation of the expD1 or expD2 deletion mutants by integration of pLM811-1 carrying the expD1 and expD2 wild-type genes into the genome of SmLM9828 (Rm2011 $\Delta$ expD1exoYmucR) or SmLM9832 (Rm2011 $\Delta$ expD2exoYmucR) also restored the ability of $S$. meliloti to produce EPS II (Table 2).

The total sugar content of the EPS fractions was determined (Table 2). A high sugar content in the culture supernatant correlated with the detection of a HMW fraction with the exception of strain SmLM9832. In the culture supernatant of this strain a LMW fraction was exclusively detected. In this LMW fraction glucose but no galactose was detected, implying that strain SmLM9832 secreted no LMW EPS II and might have secreted a high amount of cyclic glucans. In the culture supernatants of strains SmLM9828 and SmLM9836 a LMW fraction containing a low amount of total sugars was exclusively detected (Table 2 ). To confirm that the HMW fraction produced by the strains secreting ExpE1 contained EPS II, the glucose and galactose content was quantified. The HMW fractions from RmAR9007 TD101, SmLM9836::pLM93-2, SmLM9828:: pLM8111 and SmLM9832::pLM811-1 contained glucose and galactose in the ratio of approximately $1: 1$ (Table 2), indicating that these fractions contained EPS II. Glucose, but no galactose was detected in the LMW fractions of strains SmLM9828 and SmLM9836, indicating the possible presence of cyclic glucans but no LMW EPS II as was already suggested for the LMW fraction of mutant SmLM9832 (Table 2).

Since ExpE1 might function extracellularly in EPS II biosynthesis or secretion, we tested if ExpE1 could be supplied in trans to stimulate HMW-EPS II production by mutant SmLM9836. This strain, which is unable to 
produce EPS II due to the deletion of expE1 was cocultivated with SmLM8311, which secretes ExpE1 but does not produce EPS II due to a mutation in $\exp A 3$ encoding a glycosyltransferase. After $10 \mathrm{~d}$ growth in low-phosphate medium, no HMW fraction was detected in the culture supernatant (Table 2). This was also confirmed by the low sugar content in the culture supernatant.

\section{DISCUSSION}

The expE1 gene situated in the exp gene cluster of $S$. meliloti 2011 encodes the ExpE1 protein that was shown to be secreted and to bind calcium ions. These results are in accordance with similarities of ExpE1 to other secreted proteins like toxins, metalloproteases or NodO (Becker et al., 1997; Delepelaire \& Wandersman, 1990; Economou et al., 1990; Mackman et al., 1986). In addition to the $\mathrm{Ca}^{2+}$-binding domains characteristic of these proteins, they contain amphipathic $\alpha$-helices close to the $\mathrm{C}$ terminus that are thought to be involved in secretion (Duong et al., 1992, 1994; Ghigo \& Wandersman, 1992).

An amphipathic $\alpha$-helix close to the $\mathrm{C}$ terminus and 15 $\mathrm{Ca}^{2+}$-binding domains were predicted for ExpE1 (Becker et al., 1997). The repeat sequence GGXGXDXUX (where $U$ is any hydrophobic amino acid) was found in most of the members of a family of bacterial proteins that are secreted by type I secretion systems (Binet et al., 1997). The three-dimensional structure of several of these secreted proteins demonstrated that the repeated motif binds $\mathrm{Ca}^{2+}$ in a parallel $\beta$-roll structure (Baumann et al., 1993; Baumann, 1994). Baumann et al. (1993) suggested that the $\mathrm{Ca}^{2+}$-binding regions may have a role in folding of the molecule after transmembrane translocation. In the absence of $\mathrm{Ca}^{2+}$ this structure is unstable and could facilitate membrane translocation of the polypeptide in an unfolded form. The presence of $\mathrm{Ca}^{2+}$ in the extracellular medium could induce the polypeptide to fold in the right tertiary structure. Another possible role for the calcium domains was proposed for haemolysin A and the adenylate cyclase toxin from Bordetella pertussis (Bakás et al., 1998; Rose et al., 1995). In both cases, it was suggested that binding of $\mathrm{Ca}^{2+}$ may induce a conformational change that would result in surfacing hydrophobic portions of the protein. The increased hydrophobic surface probably favours cell-membrane binding that appears to be essential for the lytic process.

The $M_{\mathrm{r}}$ estimated by SDS-PAGE for ExpE1 or (His) ${ }_{6}$ ExpE1 showed an abnormal value. In both cases, this value was $8-10 \mathrm{kDa}$ higher than the $M_{\mathrm{r}}$ predicted by its amino acid sequence. The discrepancy in the predicted and observed $M_{\mathrm{r}}$ in SDS-PAGE was also reported for NodO from R. leguminosarum, a protein that shows some sequence similarity with ExpE1 (Economou et al., 1990) and other acidic proteins (Kaufman et al., 1984). Size fractionation of the (His) ${ }_{6}$ ExpE1 by gel filtration indicated that this protein purifies as a dimer. The same observation was also made for NodO (Economou et al., 1990).

$\operatorname{expD1}$ and $\operatorname{expD2}$ were required for the secretion of ExpE1. Since ExpD1 and ExpD2 are homologous to $\mathrm{ABC}$ transporters and membrane-fusion proteins, respectively, of type I secretion systems, we propose that ExpE1 is secreted by a type I secretion system involving ExpD1 and ExpD2. In a genetic background allowing strong expression of expE1, ExpE1 accumulated in the cell if $\operatorname{expD1}$ or $\operatorname{expD2}$ were deleted. Under conditions allowing a medium level expression of expE1 this was also the case in an $\operatorname{expD2}$ mutant but not in an $\operatorname{expD1}$ mutant. Since expression of an expE1-lacZ translational fusion was not affected in the expD1 deletion mutant, the low amount of ExpE1 detected might have resulted from degradation. Hence, the absence of ExpD1 may destabilize or its presence may stabilize ExpE1 in the cell. It is possible that ExpE1 was also degraded in an expD1 mutant when it was overexpressed, but due to the high amount of ExpE1 produced, this degradation might not have occurred at a relevant level.

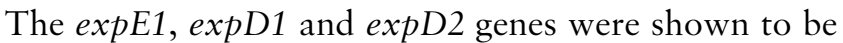
required for the synthesis or secretion of EPS II. Since secretion of ExpE1 required expD1 and expD2, ExpD1 and ExpD2 may be indirectly required for EPS II production. A direct involvement of ExpD1 and ExpD2 in EPS II production independent of their function in secretion of ExpE1 might be the secretion of EPS II or its precursors. An involvement of type I secretion systems in the secretion of polysaccharides was described for $S$. meliloti and Agrobacterium tumefaciens cyclic $(1,2)-\beta$ glucans and E. coli $\mathrm{K} 1$ capsular polysaccharide (Bliss \& Silver, 1996; Breedveld \& Miller, 1994). The NdvA and ChvA proteins from $S$. meliloti and A. tumefaciens share homologies with bacterial $\mathrm{ABC}$ transporter proteins, and mutants in these genes did not secrete cyclic $(1,2)-\beta$ glucans to the periplasmic compartment and to the extracellular medium. In E. coli $\mathrm{K} 1$, sialic acid is synthesized and polymerized in the cytoplasm, and linking of the synthetic and transport pathways is possibly performed by the proteins $\mathrm{KpsC}$ and $\mathrm{KpsS}$ that may 'escort' the polySia chains to the transport machinery. This machinery is composed of a type I secretion system comprising KpsM, KpsT and KpsE, that together with the periplasmic protein KpsD and an unknown outer-membrane protein, are responsible for the translocation of the capsular polysaccharide across the inner and outer membranes. Since ExpD1 and ExpD2 resemble components of type I protein-secretion systems and we have demonstrated that these two proteins are required for secretion of ExpE1, it appears rather unlikely that the ExpD1/ExpD2 type I transporter system is additionally involved in the secretion of a polysaccharide or polysaccharide precursors.

Supplying ExpE1 in trans in a co-cultivation experiment did not restore the ability of a S. meliloti expE1 mutant to produce extracellular EPS II. The ExpE1 protein might not be stable enough in the culture medium to correct the deficiency of an expE1 mutant in EPS II 
synthesis or to fulfil its function ExpE1 has to be synthesized and secreted by the cell that synthesizes EPS II. ExpE1 is similar to NodO from R. leguminosarum which was found to insert into liposomes and when added to planar lipid bilayers, formed cation-selective channels that allowed the movement of monovalent cations across the membrane (Sutton et al., 1994). Sutton et al. (1994) suggested that the role of NodO in nodulation might be the formation of pores in the plant plasma membrane. ExpE1 and NodO mainly consist of the nonapeptide repeats and the C-terminal 85 aa of ExpE1 display no similarities to NodO. Therefore, functions of these two proteins cannot necessarily be suggested based on these sequence similarities.

Since ExpE1 was not detected in the outer-membrane fraction, it seems to be rather unlikely that ExpE1 forms pores in this membrane that are involved in the secretion of EPS II. On the other hand, for technical reasons ExpE1 was recovered from cells and supernatants of $S$. meliloti strains that were unable to produce EPS I and EPS II. Therefore, we cannot exclude the possibility that the localization of ExpE1 in the wild-type depends on the presence of EPS I or EPS II. Weisgerber \& Troy (1990) reported that immunoprecipitated polySia obtained from labelled E. coli $\mathrm{K} 1$ membranes was found to be associated with a protein of $20 \mathrm{kDa}$ which has an unknown function. Hence, the hypothesis that ExpE1 mediates the secretion of EPS II by binding to the nascent EPS II chain might be conceivable. Although it was shown that ExpE1 is required for EPS II synthesis or secretion, the role of this protein and its transport system in EPS II production remains unknown, and further studies will be required to understand their function.

\section{ACKNOWLEDGEMENTS}

This work was supported by the TMR-Marie Curie Research Training Grant no. ERBFMBICT 97-2348 and the Human Capital and Mobility grant no. ERBCHRXCT 94-0694 from the European Community to L.M.M. A.B. acknowledges a grant from the Ministerium für Schule und Weiterbildung, Wissenschaft und Forschung des Landes Nordrhein-Westfalen in the Lise-Meitner-Programm.

\section{REFERENCES}

Ausubel, F. M., Brent, R., Kingston, R. E., Moore, D. D., Seidman, J. G., Smith J. A. \& Struhl, K. (editors) (1990). Current Protocols in Molecular Biology. Vols 1 and 2. New York: Greene Publishing and Wiley Interscience.

Bakás, L., Veiga, M. P., Soloaga, A., Ostolaza, H. \& Goñi, F. M. (1998). Calcium-dependent conformation of E. coli $\alpha$-hemolysin: implications for the mechanism of membrane insertion and lysis. Biochim Biophys Acta 1368, 225-234.

Baumann, U. (1994). Crystal structure of the $50 \mathrm{kDa}$ metalloprotease from Serratia marcescens. J Mol Biol 242, 244-251.

Baumann, U., Wu, S., Flaherty, K. M. \& McKay, D. B. (1993). Three-dimensional structure of the alkaline protease of Pseudomonas aeruginosa : a two-domain protein with a calcium binding parallel beta roll motif. EMBO J 12, 3357-3364.
Becker, A. \& Pühler, A. (1998). Production of exopolysaccharides. In The Rhizobiaceae: Molecular Biology of Model PlantAssociated Bacteria, pp. 97-118. Edited by H. P. Spaink, A. Kondorosi \& P. J. J. Hooykaas. Dordrecht: Kluwer.

Becker, A., Schmidt, M., Jäger, W. \& Pühler, A. (1995). New gentamicin resistance and lac Z promoter probe cassettes suitable for insertion mutagenesis and generation of transcriptional fusions. Gene 162, 37-39.

Becker, A., Rüberg, S., Küster, H., Roxlau, A., Keller, M., Ivashina, T., Cheng, H.-P., Walker, G. C. \& Pühler, A. (1997). The 32-kilobase exp gene cluster of Rhizobium meliloti directing the biosynthesis of galactoglucan: genetic organization and properties of the encoded gene products. J Bacteriol 179, 1375-1384.

Beringer, J. E. (1974). R factor transfer in Rhizobium leguminosarum. J Gen Microbiol 84, 188-198.

Beutler, H. O. (1984). Lactose and D-galactose: UV method. In Methods of Enzymatic Analysis, 3rd edn, vol. 6, pp. 104-112. Edited by H. U. Bergemeyer. Weinheim: Verlag Chemie.

Binet, R., Létoffé, S., Ghigo, J. M., Delepelaire, P. \& Wandersman, C. (1997). Protein secretion by Gram-negative bacterial ABCexporters: a review. Gene 192, 7-11.

Bliss, J. M. \& Silver, R. P. (1996). Coating the surface: a model for expression of capsular polysialic acid in Escherichia coli K1. Mol Microbiol 21, 221-231.

Breedveld, M. W. \& Miller, K. J. (1994). Cyclic $\beta$-glucans of members of the family Rhizobiaceae. Microbiol Rev 58, 145-161.

Bullock, W. C., Fernandez, J. M. \& Short, J. M. (1987). XL1-Blue: a high efficient plasmid transforming recA Escherichia coli strain with beta-galactosidase selection. Biotechniques 5, 376-379.

Casse, F., Boucher, C., Hulliot, J. S., Michel, M. \& Dénarié, J. (1979). Identification and characterization of large plasmids in Rhizobium meliloti using agarose gel electrophoresis. J Bacteriol 113, 229-242.

Chaplin, M. F. \& Kennedy, S. F. (1986). Carbohydrate Analysis: a Practical Approach. Washington DC: IRL Press.

Delepelaire, P. \& Wandersman, C. (1990). Protein secretion in Gram-negative bacteria: the extracellular metalloproteases from Erwinia chrysanthemi contains a C-terminal secretion signal analogous to that of Escherichia coli $\alpha$-hemolysin. J Biol Chem 265, 17118-17125.

Dinh, T., Paulsen, I. T. \& Saier, M. H., Jr (1994). A family of extracytoplasmic proteins that allow transport of large molecules across the outer membranes of gram-negative bacteria. J Bacteriol 176, 3825-3831.

Duong, F., Lazdunski, A., Cami, B. \& Murgier, M. (1992). Sequence of a cluster of genes controlling synthesis and secretion of alkaline protease in Pseudomonas aeruginosa: relationships to other secretory pathways. Gene 121, 47-54.

Duong, F., Soscia, C., Lazdunski, A. \& Murgier, M. (1994). The Pseudomonas fluorescens lipase has a C-terminal secretion signal and is secreted by a three-component bacterial ABC-exporter system. Mol Microbiol 11, 1117-1126.

Economou, A., Hamilton, W. D. O., Johnston, A. W. B. \& Downie, J. A. (1990). The Rhizobium nodulation gene nodO encodes a $\mathrm{Ca}^{2+}$-binding protein that is exported without $\mathrm{N}$-terminal cleavage and is homologous to haemolysin and related proteins. EMBO J 9, 349-354.

Fath, M. J. \& Kolter, R. (1993). ABC transporters: bacterial exporters. Microbiol Rev 57, 995-1017.

Finan, T. M., Hartwieg, E., LeMieux, K., Bergman, K., Walker, G. C. \& Signer, E. R. (1984). General transduction in Rhizobium meliloti. J Bacteriol 159, 120-124. 
Geelen, D., Van Montagu, M. \& Holsters, M. (1995). Cloning of an Azorhizobium caulinodans endoglucanase gene and analysis of its role in symbiosis. Appl Environ Microbiol 61, 3304-3310.

Ghigo, J.-M. \& Wandersman, C. (1992). Cloning, nucleotide sequence and characterization of the gene encoding the Erwinia chrysanthemi B374 PtrA metalloprotease: a third metalloprotease secreted via a C-terminal secretion signal. Mol Gen Genet 236, 135-144.

Glazebrook, J. \& Walker, G. C. (1989). A novel exopolysaccharide can function in place of the calcofluor-binding exopolysaccharide in nodulation of alfalfa by Rhizobium meliloti. Cell 56, 661-672.

Gonzáles, J. E., Reuhs, B. L. \& Walker, G. C. (1996). Low molecular weight EPS II of Rhizobium meliloti allows nodule invasion in Medicago sativa. Proc Natl Acad Sci USA 93, 8636-8641.

Harlow, E. \& Lane, D. (1988). Antibodies: a Laboratory Manual. Cold Spring Harbor, NY: Cold Spring Harbor Laboratory.

Katzen, F., Becker, A., lelmini, M. V., Oddo, C. G. \& lelpi, L. (1999). New mobilizable vectors suitable for gene replacement in Gramnegative bacteria and their use in mapping of the 3 ' end of the Xanthomonas campestris pv. campestris gum operon. Appl Environ Microbiol 65, 278-282.

Kaufman, E., Geisler, N. \& Weber, K. (1984). SDS-PAGE strongly overestimates the molecular masses of the neurofilament proteins. FEBS Lett 170, 81-84.

Keller, M., Roxlau, A., Weng, W. M., Schmidt, M., Quandt, J., Niehaus, K., Jording, D., Arnold, W. \& Pühler, A. (1995). Molecular analysis of the Rhizobium meliloti mucR gene regulating the biosynthesis of the exopolysaccharides succinoglycan and galactoglucan. Mol Plant-Microbe Interact 8, 267-277.

Kessler, C. (1992). Nonradioactive Labelling and Detection of Biomolecules. Berlin: Springer.

Kunst, A., Draeger, B. \& Zoegenhorn, J. (1984). UV methods with hexokinase and glucose-6-phosphate dehydrogenase. In Methods of Enzymatic Analysis, pp. 163-172, 3rd edn., vol. 6. Edited by H. U. Bergemeyer. Weinheim: Verlag Chemie.

Laemmli, U. K. (1970). Cleavage of structural proteins during the assembly of the head of bacteriophage T4. Nature 227, 680-685.

Leigh, J. A. \& Walker, G. C. (1994). Exopolysaccharides of Rhizobium: synthesis, regulation and symbiotic function. Trends Genet 10, 63-67.

Ludwig, A., Jarchau, T., Benz, R. \& Goebel, W. (1988). The repeat domain of Escherichia coli haemolysin (HlyA) is responsible for its $\mathrm{Ca}^{2+}$-dependent binding to erythrocytes. Mol Gen Genet 214, 553-561.

Mackman, N., Nicaud, J. M., Gray, V. \& Holland, I. B. (1986). Secretion of hemolysin by Escherichia coli. Curr Top Microbiol Immunol 125, 159-181.

Maina, C. V., Riggs, P. D., Grandea, A. G., Slatko, B. E., Moran, L. S., Tagliamonte, J. A., McReynolds, L. A. \& Guan, C. (1988). An Escherichia coli vector to express and purify foreign proteins by fusion to and separation from maltose-binding protein. Gene $\mathbf{7 4}$, 365-373.

Maruyama, K., Mikawa, T. \& Ebashi, K. (1984). Detection of calcium binding proteins by ${ }^{45} \mathrm{Ca}$ autoradiography on nitrocellulose membrane after sodium dodecyl sulfate gel electrophoresis. J Biochem 95, 511-519.

Mateos, P. F., Jimenez-Zurdo, J. I., Chen, J., Aquartini, A. S., Haack, S. K., Martinez-Molina, E., Hubbell, D. H. \& Dazzo, F. B. (1992). Cell-associated pectinolytic and cellulolytic enzymes in Rhizobium leguminosarum biovar trifolii. Appl Environ Microbiol 58, 1816-1822.

Meade, H. M., Long, S. R., Ruvkun, G. B., Brown, S. E. \& Ausubel,
F. M. (1982). Physical and genetic characterization of symbiotic and auxotrophic mutants of Rhizobium meliloti induced by transposon Tn5 mutagenesis. J Bacteriol 149, 114-122.

Miller, J. H. (1972). Experiments in Molecular Genetics. Cold Spring Harbor, NY: Cold Spring Harbor Laboratory.

Neidhardt, F. C., Bloch, P. L. \& Smith, D. F. (1974). Culture medium for enterobacteria. J Bacteriol 119, 736-747.

Poxton, I. R., Bell, G. T. \& Barclay, G. R. (1985). The association on SDS-polyacrylamide gels of lipopolysaccharide and outer membrane proteins of Pseudomonas aeruginosa as revealed by monoclonal antibodies and western blotting. FEMS Microbiol 27, 247-251.

Priefer, U. B., Simon, R. \& Pühler, A. (1985). Extension of the host range of Escherichia coli vectors by incorporation of RSF1010 replication and mobilization functions. J Bacteriol 163, 324-330.

Reinhold, B. B., Chan, S. Y., Reuber, T. L., Marra, A., Walker, G. C. \& Reinhold, V. N. (1994). Detailed structural characterization of succinoglycan, the major exopolysaccharide of Rhizobium meliloti Rm1021. J Bacteriol 176, 1997-2002.

Reuber, T. L. \& Walker, G. C. (1993). Biosynthesis of succinoglycan, a symbiotically important exopolysaccharide of Rhizobium meliloti. Cell 74, 269-280.

Rose, T., Sebo, P., Bellalou, J. \& Ladant, D. (1995). Interaction of calcium with Bordetella pertussis adenylate cyclase toxin: characterization of multiple calcium-binding sites and calcium-induced conformational changes. J Biol Chem 270, 26370-26376.

Rüberg, S., Pühler, A. \& Becker, A. (1999). Biosynthesis of the exopolysaccharide galactoglucan in Sinorhizobium meliloti is subject to a complex control by the phosphate-dependent regulator PhoB and the proteins ExpG and MucR. Microbiology 145, 603-611.

Sambrook, J., Fritsch, E. F. \& Maniatis, T. (1989). Molecular Cloning: a Laboratory Manual, 2nd edn. Cold Spring Harbor, NY: Cold Spring Harbor Laboratory.

Schäfer, A., Tauch, A., Jäger, W., Kalinowski, J., Thierbach, G. \& Pühler, A. (1994). Small mobilizable multi-purpose cloning vectors derived from the Escherichia coli plasmids pK18 and pK19: selection of defined deletions in the chromosome of Corynebacterium glutamicum. Gene 145, 69-73.

Schirmer, F., Ehrt, S. \& Hillen, W. (1997). Expression, inducer spectrum, domain structure, and function of MopR, the regulator of phenol degradation in Acinetobacter calcoaceticus NCBI 8250. J Bacteriol 179, 1329-1336.

Sharypova, L. A., Yurgel, S. N., Keller, M., Simarov, B. V., Pühler, A. \& Becker, A. (1999). The eff-482 locus of Sinorhizobium meliloti CXM1-105 that influences symbiotic effectiveness consists of three genes encoding an endoglycanase, a transcriptional regulator and an adenylate cyclase. Mol Gen Genet 261, 1032-1044.

Simon, R., Priefer, U. \& Pühler, A. (1983). A broad-host-range mobilization system for in vivo genetic engineering: transposon mutagenesis in gram-negative bacteria. Biotechnology 1, 784-791.

Sutton, J. M., Lea, E. J. A. \& Downie, J. A. (1994). The nodulationsignalling protein NodO from Rhizobium leguminosarum biovar viciae forms ion channels in membranes. Proc Natl Acad Sci USA 91, 9990-9994.

Towbin, H., Staehelin, T. \& Gordon, J. (1979). Electrophoretic transfer of proteins from polyacrylamide gels to nitrocellulose sheets: procedure and some applications. Proc Natl Acad Sci USA 76, 4350-4354.

Wang, L.-X., Wang, Y., Pellock, B. \& Walker, G. C. (1999). Structural characterization of the symbiotically important low- 
molecular-weight succinoglycan of Sinorhizobium meliloti. J Bacteriol 181, 6788-6796.

Weigerber, C. \& Troy, F. A. (1990). Biosynthesis of the polysialic acid capsule in Escherichia coli K1: the endogenous acceptor of polysialic acid is a membrane protein of $20 \mathrm{kDa}$. J Biol Chem $\mathbf{2 6 5}$, 1578-1587.

York, G. M. \& Walker, G. C. (1997). The Rhizobium meliloti exoK gene and $p r s D / p r s E /$ exsH genes are components of independent degradative pathways which contribute to production of lowmolecular-weight succinoglycan. Mol Microbiol 25, 117-134.
Zhan, H., Levery, S. B., Lee, C. C. \& Leigh, J. A. (1989). A second exopolysaccharide of Rhizobium meliloti strain SU47 that can function in root nodule invasion. Proc Natl Acad Sci USA 86, 3055-3059.

Zhan, H., Lee, C. C. \& Leigh, J. A. (1991). Induction of the second exopolysaccharide (EPSb) in Rhizobium meliloti strain SU47 by low phosphate concentrations. J Bacteriol 173, 7391-7394.

Received 12 May 2000; accepted 19 May 2000. 